Published in final edited form as:

Med Case Rep (Wilmington). 2017 ; 3(2): . doi:10.21767/2471-8041.100044.

\title{
Bilateral Uveitis and Keratitis Following Nivolumab Treatment for Metastatic Melanoma
}

\author{
Douglas M Baughman ${ }^{1}$, Cecilia S Lee ${ }^{1}$, Brian E Snydsman ${ }^{2}$, and Hoon C Jung ${ }^{1,2}$ \\ ${ }^{1}$ Department of Ophthalmology, University of Washington, Seattle, Washington, USA \\ ${ }^{2}$ Veterans Affairs Puget Sound Health Care System, Seattle, Washington, USA
}

\begin{abstract}
Background-Inflammatory eye disease has been reported as a side effect with Nivolumab.

Case presentation-We report a case of a 92-year-old woman presenting with bilateral and simultaneous keratitis and uveitis in the setting of recurring infusions of nivolumab for metastatic melanoma. The patient underwent successful coordinated treatment of both eyes coinciding with ongoing systemic infusion treatments with ophthalmic topical medications alone.

Conclusion-The interest of this case resides in the simultaneous nature of presentation of eye inflammation both internally and of the ocular surface. Prior case reports have cited uveitis or ocular surface disease, however not in simultaneous fashion. Clinicians should raise their index of suspicion of side effects of nivolumab systemic infusion for any vision or eye symptom changes around the timing of treatment.
\end{abstract}

\section{Keywords}

Nivolumab; Uveitis; Keratitis

\section{Introduction}

Nivolumab is an anti-programmed cell death protein-1 (anti-PD-1) monoclonal antibody in an emerging class of anti-tumor therapies inhibiting T-cell immune checkpoints [1].

Nivolumab is approved by the Food and Drug Administration (FDA) for treatment of patients with non-surgical or metastatic melanoma, metastatic non-small cell lung cancer, renal cell carcinoma, classical Hodgkin's lymphoma, squamous cell carcinoma of the head and neck, and urothelial carcinoma [2]. Published case reports have cited development of either secondary uveitis or ocular surface disease, separately, associated with use of the

\footnotetext{
Corresponding author: Hoon C. Jung, Veterans Affairs Puget Sound Health Care System, 1660 S. Columbian Way, Eye Clinic, Seattle, WA 98108, USA, Tel: 207-277-2020; Hoon.jung2@va.gov.

Authors' Contributions

Douglas M Baughman and Hoon C Jung wrote the case report., Cecilia S Lee and Brian E Snydsman reviewed the manuscript and corrected it. All authors read and approved the final manuscript.

Consent for Publication

Consent for publication in written form from the local IRB was obtained.
} 
medication [3-6]. We present this as a case of bilateral and simultaneous keratitis and uveitis in the setting of nivolumab infusions for metastatic melanoma.

\section{Case Presentation}

A 92-year-old woman presented with bilateral blurred vision after her third infusion of nivolumab as monotherapy for metastatic BRAF positive malignant melanoma. The patient reported progressive vision decrease over the past 1.5 weeks without pain, redness or light sensitivity. She self-reported a history of normal eye exams other than age related lens changes diagnosed at a separate facility in the past year. The patient used artificial tears on occasion. The patient had no family history of glaucoma, macular degeneration or other blinding conditions and had no known medication allergies. Best corrected visual acuity (BCVA) was 20/250 for the right eye and 20/200 for the left eye using Snellen acuity chart testing. Additional findings included severe superficial keratitis of both eyes with epithelial irregularity, sub epithelial haze and find endothelial deposits left eye greater than right eye. The anterior chamber had 1+ flare with trace cell in each eye. Posterior synechiae were present at focal areas in each eye with $3+$ nuclear sclerotic lens changes in both eyes (Figure 1). The posterior exam was normal in the context of the limited view. Optical coherence tomography (OCT) of the macula for each eye revealed no macular edema. Topical prednisolone acetate $(1 \%)$, cyclopentoloate $(1 \%)$ dilating drops tobramycin $(0.3 \%) /$ dexamethasone $(0.1 \%)$ ophthalmic ointment and carboxymethylcellulose (1\%) artificial tears were initiated as primary therapy. Three days later BCVA improved to 20/150-1 in the right eye and 20/80-1 in the left eye with improvement of previous exam findings. Given the rapid therapeutic response to topical therapy and need for ongoing systemic cancer therapy, a plan of pulsing topical steroids to six times daily one day prior to nivolumab infusion with a taper and follow up eye exam the same week of the infusion was initiated. This modality of therapy was used with each subsequent cycle of systemic therapy. Within two weeks BCVA had improved to 20/50 in each eye limited by cataract. Of note posterior synechiae remained despite topical eye therapy. Three months of nivolumab infusion therapy was successfully administered while maintaining stability of the vision and eye findings at a steady state with the above mentioned topical regimen. At the end of this course the patient had reported decrease in size of the known metastatic lesions in the left axillary node, however with an increase in size of the supraclavicular node. The patient was converted to vemurafenib oral therapy according to medical decisions independent from the ocular course.

\section{Discussion}

The discussion of cancer immuno-editing elucidated the role of the immune system in surveillance and destruction of nascent tumor cells [7]. Soon after, monoclonal antibodies directed against the co-inhibitory immune checkpoint molecule PD-1 were developed to augment the immune function of detecting and destroying cancer cells [1]. This disruption of immune checkpoint molecules alters host immune tolerance and introduces a new array of side effects termed the immune-related adverse events (irAEs), which manifest as autoimmune-like inflammatory disease [8]. In nivolumab, the most common irAEs seen in Phase I-III clinical trials were fatigue, pulmonary disease, skin rash, and endocrinopathies 
[8]. Uveitis was reported in $0.4 \%$ of patients during a phase III trial of pemrbolizumab, another anti-PD-1 monoclonal antibody [9]. Anterior uveitis emerged as an irAE in several case reports of patients receiving nivolumab and has responded to topical or intraocular steroids in each case [3-5].

Dry eye disease associated with nivolumab was seen during a phase I clinical trial in 2 of 207 patients [10]. A recent case report showed progression of dry eye disease to corneal perforation in a patient who did not discontinue nivolumab at initial presentation. Eye symptoms were subsequently stabilized using topical medications with adjunct aggressive lubrication [6]. To our best knowledge, this is the first case of a patient with bilateral anterior uveitis and keratitis to be reported. Topical steroid therapy may be helpful in decreasing ocular symptoms during nivolumab infusions.

\section{Conclusion}

Recognizing the potential for ophthalmic side effects and complications deserves additional attention as newer immune system modulating therapies are included in the treatment of diseases. Planned coordination of efforts in the context of multi-specialty care may provide the best avenues for diagnosing and managing of primary pathology and containment of side effects from the treatment itself.

\section{Acknowledgments}

Unrestricted grant from the Research to Prevent Blindness (RPB).

Funding

Grant Information: NEI K23EY02492 (Cecilia S. Lee, MD, MS).

\section{References}

1. Rajan A, Kim C, Heery CR, Guha U, Gulley JL. Nivolumab, anti-programmed death-1 (PD-1) monoclonal antibody immunotherapy: Role in advanced cancers. Hum Vaccin Immunother. 2016; 12:2219-2231. [PubMed: 27135835]

2. Product Information: OPDIVO(R) IV injection, nivolumab IV injection. Bristol-Myers Squibb Company (per FDA); Princeton, NJ: 2017.

3. Karlin J, Gentzler R, Golen J. Bilateral anterior uveitis associated with nivolumab therapy. Ocul Immunol Inflamm. 2016:1-3.

4. De Velasco G, Bermas B, Choueiri TK. Autoimmune arthropathy and uveitis as complications of programmed death 1 inhibitor treatment. Arthritis Rheumatol. 2016; 68:556-557. [PubMed: 26314277]

5. Arai T, Harada K, Usui Y, Irisawa R, Tsuboi R. Case of acute anterior uveitis and Vogt-KoyanagiHarada syndrome-like eruptions induced by nivolumab in a melanoma patient. J Dermatol. 2016

6. Nguyen AT, Elia M, Materin MA, Sznol M, Chow J. Cyclosporine for dry eye associated with nivolumab: A case progressing to corneal perforation. Cornea. 2016; 35:399-401. [PubMed: 26771550]

7. Dunn GP, Bruce AT, Ikeda H, Old LJ, Schreiber RD. Cancer immunoediting: From immunosurveillance to tumor escape. Nat Immunol. 2002; 3:991-998. [PubMed: 12407406]

8. Naidoo J, Page DB, Li BT, Connell LC, Schindler K, et al. Toxicities of the anti-PD-1 and anti-PDL1 immune checkpoint antibodies. Ann Oncol. 2015; 26:2375-2391. [PubMed: 26371282] 
9. Robert C, Schachter J, Long GV, Arance A, Grob JJ, et al. Pembrolizumab versus Ipilimumab in Advanced Melanoma. N Engl J Med. 2015; 372:2521-2532. [PubMed: 25891173]

10. Brahmer JR, Tykodi SS, Chow LQ, Hwu WJ, Topalian SL, et al. Safety and activity of anti-PD-L1 antibody in patients with advanced cancer. N Engl J Med. 2012; 366:2455-2465. [PubMed: 22658128] 


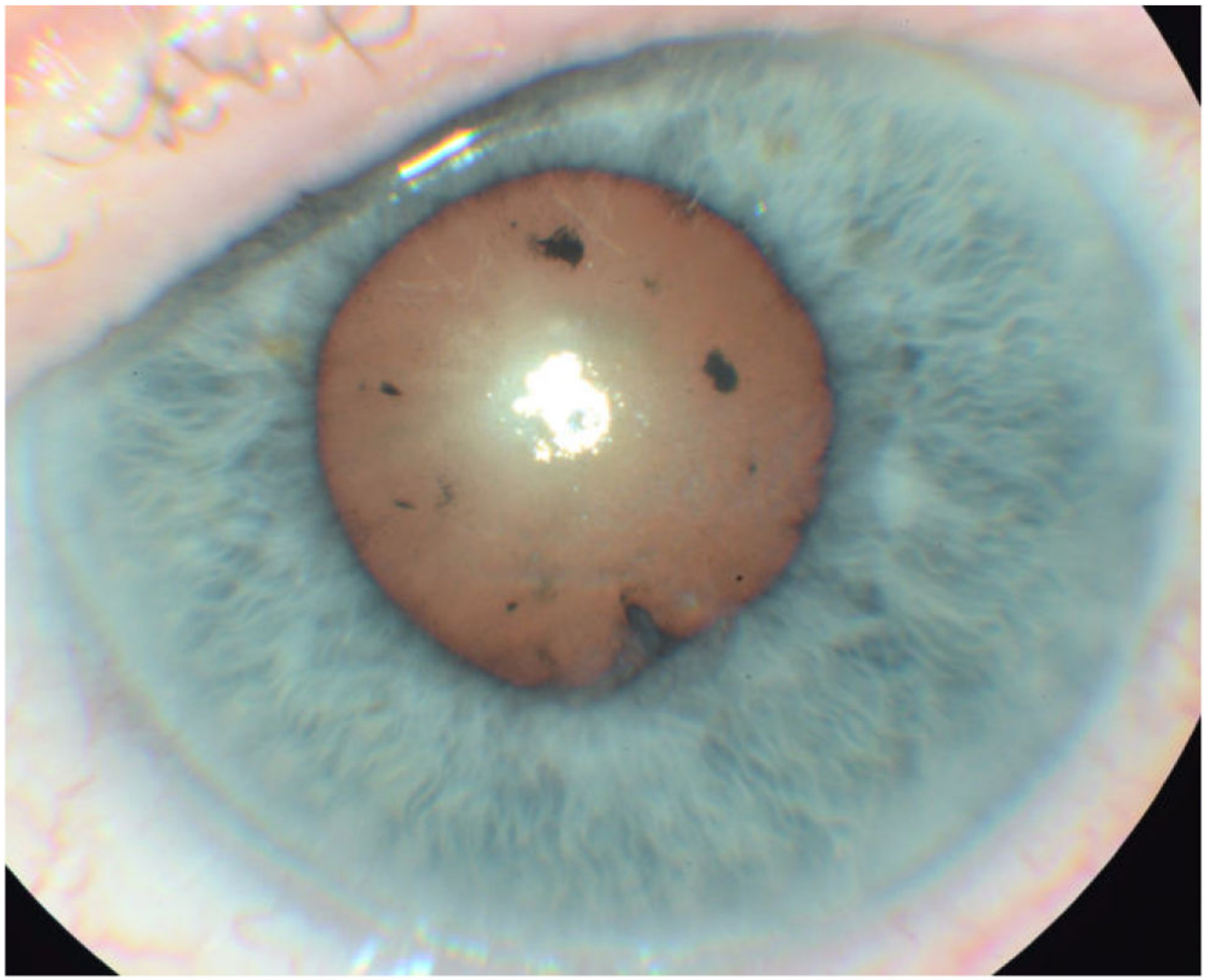

Figure 1.

Slit lamp photo of the left eye 10 days after presentation with topical therapy initiation demonstrating uveitis associated posterior synechiae and glistening ocular surface from punctate corneal irregularities. 